\title{
Germanium implanted Bragg gratings in Silicon on Insulator waveguides
}

\author{
Renzo Loiacono ${ }^{\mathrm{a}^{*}}$, Graham T. Reed ${ }^{\mathrm{a}}$, Russell Gwilliam ${ }^{\mathrm{a}}$, Goran Z. Mashanovich ${ }^{\mathrm{a}}$, Liam \\ O'Faolain $^{\mathrm{b}}$, Thomas Krauss ${ }^{\mathrm{b}}$, Giorgio Lulli ${ }^{\mathrm{c}}$, Chris Jeynes ${ }^{\mathrm{a}}$, and Richard Jones ${ }^{\mathrm{d}}$ \\ ${ }^{a}$ Advanced Technology Institute, University of Surrey, Guildford, Surrey, GU2 7XH, UK \\ ${ }^{\mathrm{b}} \mathrm{Sch}$ ool of Physics and Astronomy, North Haugh, St Andrews, Fife, KY16 9SS, UK \\ 'Istituto per la Microelettronica e Microsistemi, via Piero Gobetti, 101, 40129 Bologna, \\ Italy \\ ${ }^{\mathrm{d}}$ Intel Corporation, Photonic Technology Laboratories, SC12-326, 2200 Mission College \\ Boulevard, Santa Clara, CA USA 95054
}

\begin{abstract}
Integrated Bragg gratings are an interesting candidate for waveguide coupling, telecommunication applications, and for the fabrication of integrated photonic sensors. These devices have a high potential for optical integration and are compatible with CMOS processing techniques if compared to their optical fibre counterpart.

In this work we present design, fabrication, and testing of Germanium ion implanted Bragg gratings in silicon on insulator (SOI). A periodic refractive index modulation is produced in a $1 \mu \mathrm{m}$ wide SOI rib waveguide by implanting Germanium ions through an $\mathrm{SiO}_{2}$ hardmask.

The implantation conditions have been analysed by 3D ion implantation modelling and the induced refractive index change has been investigated on implanted samples by Rutherford Backscattering Spectroscopy (RBS) and ellipsometry analysis.

An extinction ratio of up to $30 \mathrm{~dB}$ in transmission, around the $1.55 \mu \mathrm{m}$ wavelength, has been demonstrated for Germanium implanted gratings on SOI waveguides.
\end{abstract}

Keywords: Defect engineering, Bragg Gratings, Silicon Photonics, Optical Filters.

\section{INTRODUCTION}

Silicon photonics is the response to the challenges of the telecommunication markets to increasing performance at modest costs. The integration of micro and nanophotonic devices on the traditional CMOS platform promises to overcome the limitations of traditional silicon electronics [1].

Integrated gratings are an interesting candidate as waveguide couplers [2] and optical filters [3], as well for the fabrication of integrated photonics sensors [4]. Etched SOI Bragg gratings have been successfully demonstrated in recent years [5], although there are currently no commercial applications using silicon waveguide Bragg gratings. This is due to the fact that Bragg waveguide devices in silicon are relatively new and most of the available applications rely on comparatively cheap and easy to fabricate fibre gratings. On the other hand, employing waveguide configurations would mean a higher potential for optical integration and compatibility with CMOS processing techniques. Furthermore, as the requirements for optical integration become more demanding, new fabrication techniques are investigated in order to improve device performance and cost effectiveness. As an example laser written Bragg gratings on porous silicon have been recently reported [6], and also silicon implanted Bragg gratings have been recently demonstrated by Bulk et al [7].

By using established ion implantation processes it is possible to introduce structural modifications in the crystalline lattice [8], in order to obtain a refractive index change suitable for grating fabrication. A periodic pattern can be transferred on a silicon waveguide by implanting group IV ions through a $\mathrm{SiO}_{2}$ hardmask used as template.

*corresponding author: r.loiacono@surrey.ac.uk

Silicon Photonics V, edited by Joel A. Kubby, Graham T. Reed, Proc. of SPIE Vol. 7606, $76060 \mathrm{G}$

(c) 2010 SPIE $\cdot$ CCC code: $0277-786 X / 10 / \$ 18 \cdot$ doi: $10.1117 / 12.839502$ 
A Bragg grating produced by this technique offers CMOS compatibility and fabrication flexibility for planar devices. Moreover its performance has been demonstrated to be comparable to an etched device [9].

The aim of this work is to design, fabricate, and characterize an integrated waveguide Bragg grating produced by ion implantation and defect engineering into Silicon.

\section{DEVICE DESIGN}

The device concept is shown in figure 1. A masking layer of $\mathrm{SiO}_{2}$ is deposited on a silicon on insulator rib waveguide. The grating pattern is subsequently defined in the oxide surface by e-beam lithography and etched by a reactive ion etching process. The devices are subsequently implanted with Germanium ions.

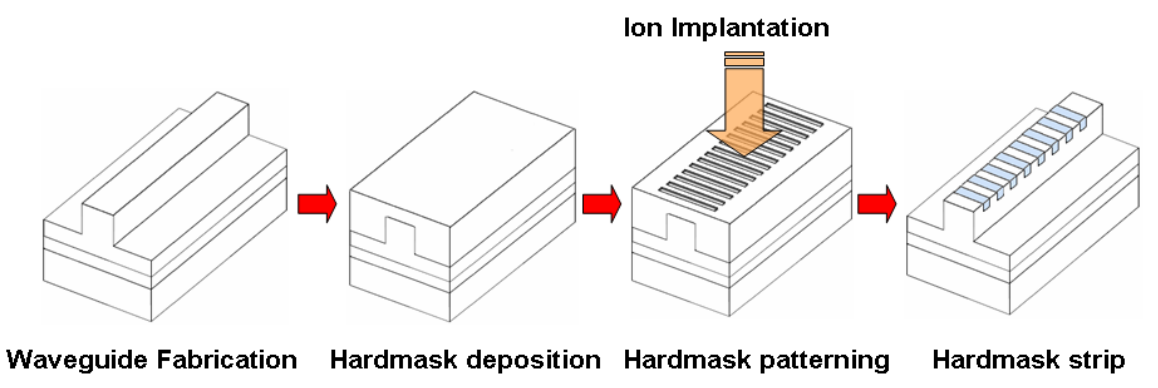

Fig. 1. Schematic representation of the masking and implantation process.

Germanium ions have been chosen in order to achieve the refractive index modulation necessary for the Bragg grating. This ion specie allows the formation of higher refractive index amorphous material at relatively low dose $\left(10^{15}\right.$ ions $\left./ \mathrm{cm}^{2}\right)$.

The reflectivity $(R)$ of a periodic optical medium satisfying the Bragg condition can be expressed by coupled mode theory analysis [10]

$$
R=\frac{\kappa^{*} \kappa \sinh ^{2}(s L)}{s^{2} \cosh ^{2}(s L)+\left(\frac{\Delta \beta}{2}\right) \sinh ^{2}(s L)}
$$

where $\kappa$ is the efficiency of the grating coupling, $L$ is the grating length, and $s$ and $\Delta \beta$ are related to the phase matching condition between transmitted and reflected light.

The coupling constant $\kappa$ is expressed as [10]

$$
\kappa=\frac{k_{0}}{4 n_{\text {eff }}} \frac{\iint_{\text {grating }} \sin \left(\mathrm{m}_{\mathrm{C}} \mathrm{c}\right) \Delta n^{2} E^{2} d x d y}{\iint_{0}^{\infty} E^{2} d x d y}
$$

where $k_{0}$ is the wavevector of the propagating light in the vacuum $\Delta n$ is the refractive index difference between each grating section, $D_{c}$ is the grating duty cycle (defined as $\xi / \Lambda$, where $\xi$ is the implanted fraction of the grating period $\Lambda$ ), $E$ is the transverse electric field amplitude, and $m$ is the grating order.

The phase matching condition is expressed as

$$
\Lambda=m \frac{\lambda c}{2 n_{e f f}}
$$


$\lambda_{c}$ being the central wavelength of the grating stop band, $n_{\text {eff }}$ the effective index of the propagating mode and $\Lambda$ the grating period.

It is assumed that the depth of the grating is given by the thickness of the amorphous layer formed by ion implantation. The influence of ion implantation on the optical properties of Silicon has been investigated by many authors. Most notably Baranaova [8] investigated the effects of silicon irradiation by different ion species, by relating the ion mass of the implanted species to the dose necessary to amorphise silicon and by investigating the subsequent refractive index change. Many authors have reported the threshold for amorphization as a concentration of point defects of approximately $10^{22} \mathrm{~cm}^{-3}$ [11]. We therefore assume that the material is completely amorphous when the concentration of point defects reaches a value of $4 \times 10^{22} \mathrm{~cm}^{-3}$, which corresponds to $80 \%$ of the crystalline Silicon atomic density.

The single mode condition has been investigated by using Photon Design software Fimmwave [12]. The waveguide dimensions have been chosen in order to achieve single mode operation and low modal birefringence. Figure 2 shows the dimensions used for the rib waveguide. The total silicon EPI thickness is $\mathrm{H}=$ $1.3 \mu \mathrm{m}$, the slab height is $\mathrm{D}-\mathrm{H}=0.6 \mu \mathrm{m}$, rib width is $\mathrm{W}=1 \mu \mathrm{m}$, and the buried oxide thickness is $1 \mu \mathrm{m}$.

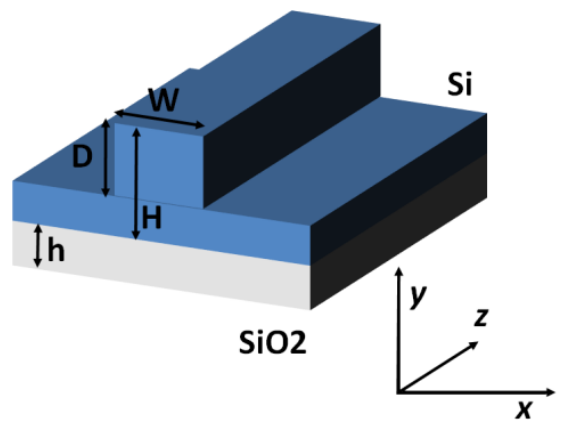

Figure 2. Proposed Silicon on Insulator rib waveguide structure

The data extracted from the ion implantation experiments described in section 3 has been used to account for the refractive index change in silicon. The coupling constants for the implanted gratings have been calculated by assuming an ideal rectangular profile, whereas the ion implantation straggling has been taken into account by assuming an equivalent increased duty cycle based on the ion implantation damage simulations presented in the next section.
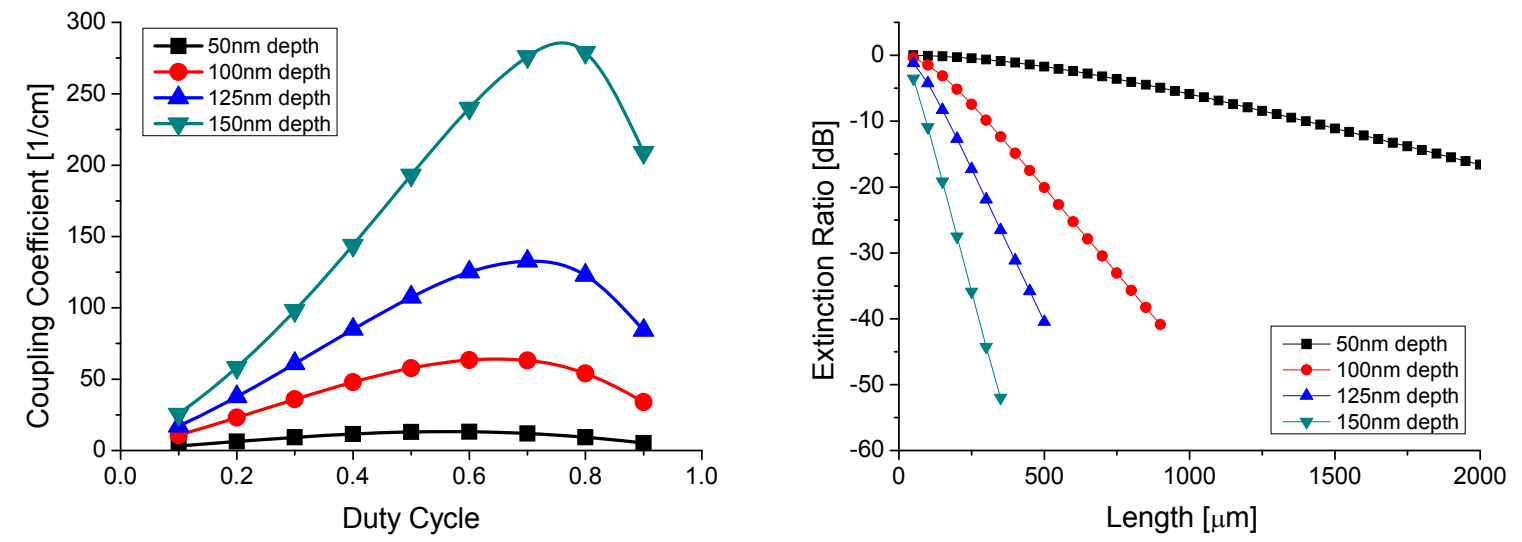

Figure 3. Calculated coupling efficiency vs. different values of duty cycle for different values of grating depth. Calculated extinction ratio in transmission vs. grating length for different grating depth.

Grating depths between $50 \mathrm{~nm}$ and $150 \mathrm{~nm}$ were simulated. Grating lengths were chosen between $250 \mu \mathrm{m}$ and $2 \mathrm{~mm}$. The aim of the design was to obtain an extinction ratio of not less than $20 \mathrm{~dB}$. Figure 3-a shows the coupling efficiency constants calculated by using the Coupled Mode Theory approximation. Assuming that in 
the ideal case the grating duty cycle is $50 \%$, figure 3 -b shows how the extinction ratio varies in the four different implantation cases for gratings lengths up to $2 \mathrm{~mm}$.

\section{ION IMPLANTATION CONDITIONS}

Ion implantation for grating patterning has been modelled by the 3D version of the Monte-Carlo binary collision approximation (MC-BCA) program KING [13]. The ion implantation induced refractive index change in silicon has been investigated by ellipsometry analysis paired with Rutherford backscattering (RBS) spectroscopy.

Figure 4 shows the damage distribution cross section in the $y, z$ plane for a single grating element for four implanted energies between $30 \mathrm{keV}$ and $90 \mathrm{keV}$, corresponding to the four grating depths presented in the previous section.

Two additional factors influencing the grating performance are introduced by the ion implantation process. The first one is ion straggling, which renders the grating profile non rectangular. The second one is the optical loss, which also limits the extinction ratio of the grating response.

Figure 4 shows a comparison between different ion implantation energies through a single hardmask slit with $115 \mathrm{~nm}$ width, corresponding to a single grating period with $50 \%$ duty cycle. The central area displayed in the pictures is assumed to be completely amorphous material (damage level $80 \%-90 \%$ ), and its lateral extension below the hardmask boundary allows quantification of the influence of the ion straggling on the grating duty cycle. We consider the amorphous to crystalline interface region as the area in which the damage decreases from $80 \%$ to $10 \%$. As an example, by simulating ion implantation of $10^{15}$ ions $/ \mathrm{cm}^{2}$ on a $230 \mathrm{~nm}$ period mask with $50 \%$ duty cycle we obtain a maximum lateral straggling of $16.5 \mathrm{~nm}$ on each side for the $70 \mathrm{keV}$ implant and of $23.5 \mathrm{~nm}$ for the $90 \mathrm{keV}$ implant. This means that we obtain an equivalent duty cycle of $64 \%$ in the first case, and an equivalent duty cycle of $70 \%$ in the second case.

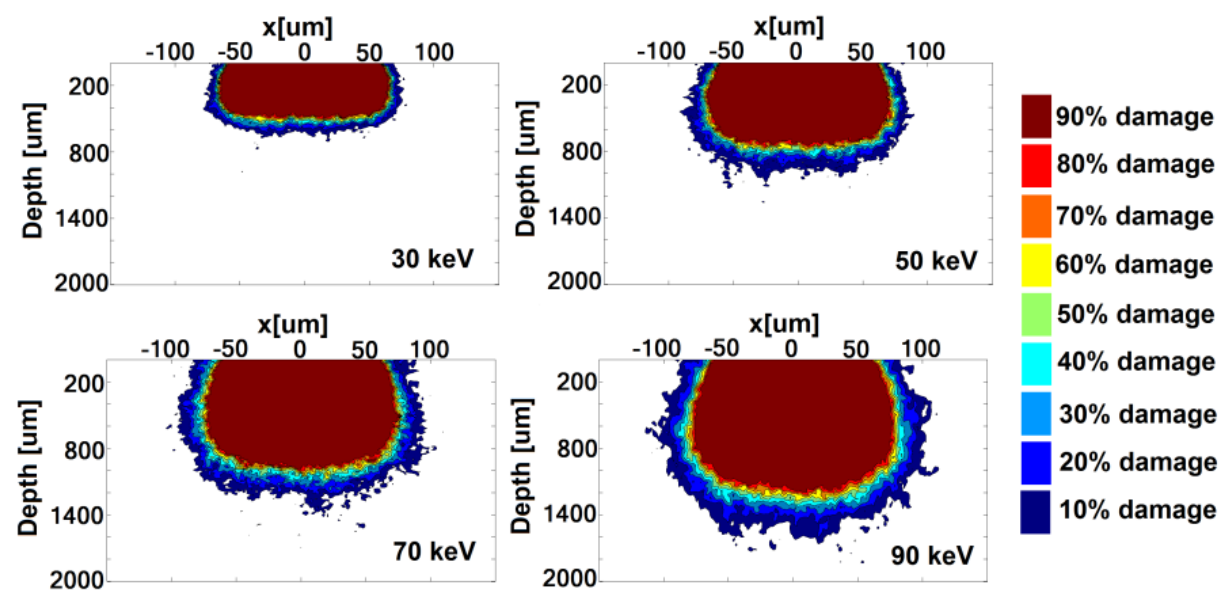

Fig. 4. Damage distribution simulation obtained by KING [13] representing Ge ions implanted through a $115 \mathrm{~nm}$ wide aperture for energies varying between $30 \mathrm{keV}$ and $90 \mathrm{keV}$. The completely amorphous region is shown as the area containing a value of displaced atoms between $80 \%$ and $90 \%$ with respect to the silicon atomic density.

Figure 5 shows the real part of the measured refractive indices for Ge amorphised silicon. The refractive index change measured proved to be suitable for grating design. The refractive index change measured is consistent with what has been previously observed by Knights et al [14] for self implants in Silicon.

The ellipsometry measurements showed that germanium implants at $10^{15}$ ions $/ \mathrm{cm}^{2}$ lead to a refractive index change of 0.5 at the $1.55 \mu \mathrm{m}$ wavelength. In order to minimize the number of variables in the ellipsometry data fitting, the samples have been preemptively analyzed with Rutherford Backscattering Spectroscopy in order to obtain an estimation of the amorphous region depth. A Tauc-Lorentz oscillator model has been used for the data fitting [15]. 


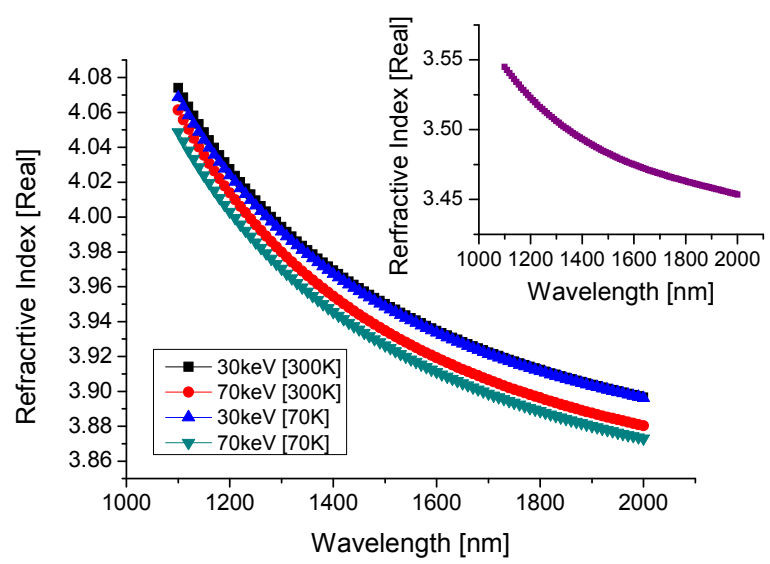

Fig. 5. Ellipsometry measurements on bulk implanted samples. The graph shows that it is possible to achieve a refractive index increase of approximately 0.5 by implanting Ge ions either at low temperature (LT - 70K) or room temperature (RT $300 \mathrm{~K})$. The inset shows data for unimplanted silicon [16].

\section{DEVICE FABRICATION}

Rib waveguides have been fabricated by UV lithography and reactive ion etching on a SOI wafer, using the dimensions described in section 2. After waveguide etching the structures have been passivated with a $300 \mathrm{~nm}$ layer of $\mathrm{SiO}_{2}$ and subsequently planarized. The oxide layer would later be used as hardmask.

The samples covered by the oxide hardmask have been spin coated with ZEP520 electron beam resist, and the the grating patterns were defined using a hybrid ZEISS GEMINI 1530/RAITH ELPHY PLUS electron beam writer. The grating period chosen was $\Lambda=230 \mathrm{~nm}$ and the grating lengths varied between $250 \mu \mathrm{m}$ and $2 \mathrm{~mm}$. The patterns have been subsequently etched in the hardmask by using an RIE process with a mixture of $\mathrm{CHF}_{3}$ and Ar. Figure 6 shows an SEM image of the implanted hardmask with a 230nm period.

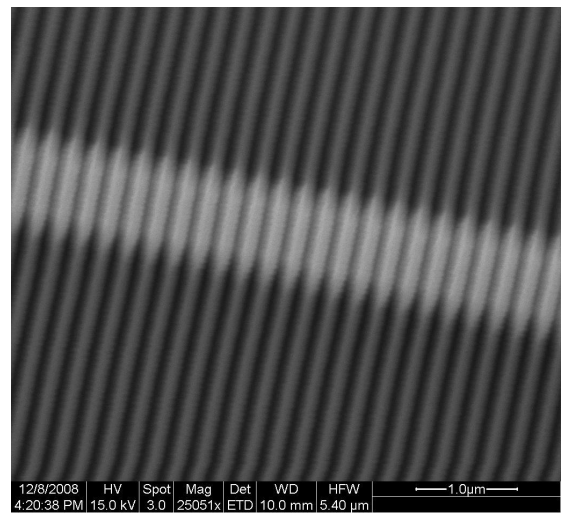

Fig. 6. $230 \mathrm{~nm}$ period hardmask fabricated for ion implantation. The rib waveguide is visible in brighter color.

The chips have been subsequently implanted with Germanium ions. Implants have been carried out at low temperature as a cautionary measure. Samples have been implanted with a dose of $10^{15}$ ions $/ \mathrm{cm}^{2}$ at an energy of $90 \mathrm{keV}$. After ion implantation the chip facets have been polished in order to ensure efficient light coupling into the chip.

\section{EXPERIMENTAL RESULTS}

Implanted devices have been optically tested by using an Agilent 8194A tunable laser source at wavelengths between $1540 \mathrm{~nm}$ and $1560 \mathrm{~nm}$, and an Agilent $816940 \mathrm{~A}$ as photodetector. Figure 7 shows the transmission 
measurements for the $90 \mathrm{keV}$ implanted devices for lengths between $250 \mu \mathrm{m}$ and $2 \mathrm{~mm}$. These devices display an extinction ratio up to $30 \mathrm{~dB}$ and a full width half maximum bandwidth up to $1.4 \mathrm{~nm}$.

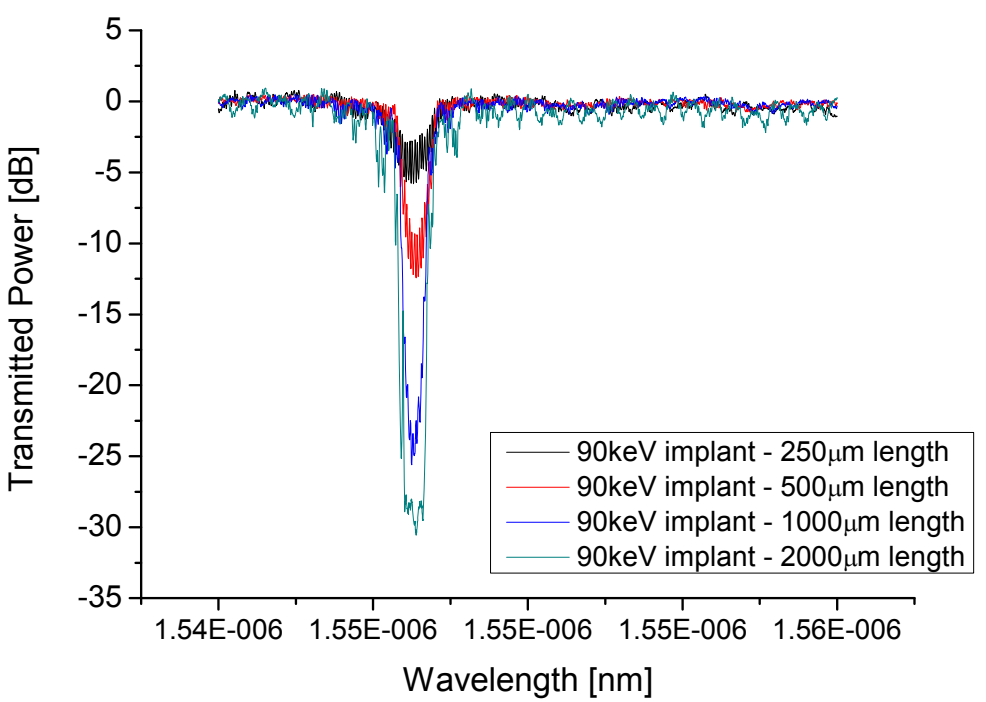

Figure 7. Grating frequency response in transmission for $90 \mathrm{keV}$ implanted gratings (a), and 70keV implanted gratings (b).

Grating losses have been evaluated by the cutback method. The $90 \mathrm{keV}$ implanted devices have shown an average loss of $12 \mathrm{~dB} / \mathrm{cm}$.

The average coupling constant evaluated for the characterized devices is $50 \pm 20 \mathrm{~cm}^{-1}$, which differ by a factor of almost 3 from the theoretical coupling constant showed in section 2. The variation found in the coupling constant evaluation at different length is attributed to fabrication tolerances in the grating patterning and is currently under investigation.

\section{CONCLUSION}

An extinction ratio up to $30 \mathrm{~dB}$ in transmission has been successfully demonstrated for Germanium implanted Bragg gratings in Silicon on Insulator rib waveguides. The measured coupling constants appear to be approximately three times lower than those initially predicted. This discrepancy can be attributed to the non ideal profile introduced by the ion implantation, and to the non uniformities between each fabricated device, such as stitching errors. The limitations in grating fabrication are currently under investigation.

Compared to previous work on implanted gratings, the choice of implantation conditions shows that heavier ions tend to produce a more self contained damage distribution, limiting the straggling effect below the mask.

Furthermore, ions heavier than silicon allow a reduction of the temperature control in the process, permitting room temperature implants, as shown by ellipsometry measurements.

\section{ACKNOWLEDGMENT}

Renzo Loiacono, Graham T. Reed, Russell Gwilliam, and Goran Z. Mashanovich would like to thank Intel Corporation for the support with waveguide fabrication and sample preparation and for financial support to the project, and IeMRC (UK) for financial support to the project. 


\section{REFERENCES}

[1] Reed, G.T.(ed), "Silicon Photonics: The State of the Art”, WileyBlackwell, (2008)

[2] Masanovic, G.Z., et al. "Preliminary experimental results of a dual grating-assisted directional coupler on SOI," Proceedings of SPIE 5730, pp. 173-180 (2005)

[3] Cohen, O., et al. "SOI-based monolithic integration of SiON and Si planar optical circuits, " United States: International Society for Optical Engineering, (2006)

[4] Passaro, V.M.N., et al., "Design of Bragg grating sensors based on submicrometer optical rib waveguides in SOI," IEEE Sensors Journal 8(9), p. 1603-11 (2008)

[5] Murphy, T.E., J.T. Hastings, and H.I. Smith, "Fabrication and characterization of narrow-band Bragg-reflection filters in silicon-on-insulator ridge waveguides, "Journal of Lightwave Technology 19(12), p. 1938-42 (2001)

[6] Marino, A., et al. "Laser direct-writing of Bragg gratings waveguides on porous silicon," 2008 International Conference on Advanced Optoelectronics and Lasers, p. 177-9 (2008)

[7] Bulk, M.P., et al., "Optical Filters Utilizing Ion Implanted Bragg Gratings in SOI Waveguides," Advances in Optical Technologies, (2008)

[8] Baranova, E.C., et al., "On silicon amorphization during different mass ion implantation," Radiation Effects 18, p. 21-26 (1973)

[9] Bulk, M.P., "Ion implanted Bragg gratings in SOI rib waveguides, " (Master Degree Thesis) McMaster University, (2008)

[10] Yariv, A. and P. Yeh, "Optical Waves in Crystal” John Wiley \& Sons, (1983)

[11] Hobler, G. and G. Otto, "Status and open problems in modeling of as-implanted damage in silicon," Materials Science in Semiconductor Processing 6, p. 1-14 (2003)

[12] Fimmwave by Photon Design, http://www.photond.com/, (2007)

[13] Lulli, G., et al., "Stopping and damage parameters for Monte Carlo simulation of MeV implants in crystalline Si," Journal of Applied Physics 82(12), p. 5958 (1997)

[14] Knights, A.P., et al., "Modification of silicon waveguide structures using ion implantation induced defects, " Applied Surface Science 255(1), p. 75-77 (2008)

[15] Jellison, G.E., Jr. and F.A. Modine, "Parameterization of the optical functions of amorphous materials in the interband region," Applied Physics Letters 69(3), p. 371-3 (1996)

[16] Frey, B.J., D.B. Leviton, and T.J. Madison, "Temperature-dependent refractive index of silicon and germanium," USA: SPIE - The International Society for Optical Engineering, (2006) 